L. da Silva, M. Spite, J. R. de Medeiros, eds.

\title{
White Dwarf Probes of Interstellar Deuterium
}

\author{
Wayne Landsman \\ Raytheon ITSS, Code 681, NASA/GSFC, Greenbelt, MD 20771
}

\begin{abstract}
.
We review the advantages of using hot white dwarfs (WDs) as probes of the deuterium abundance in the local interstellar medium. We then discuss advantages of the Space Telescope Imaging Spectrograph (STIS) for such observations, as compared with earlier observations with the Goddard High Resolution Spectrograph (GHRS). The GHRS Ly $\alpha$ profile of the white dwarf $\mathrm{HZ} 43$ is probably modified by the hot "hydrogen wall" surrounding the Sun; but despite this complication, the sightline remains a promising one for an accurate determination of the deuterium abundance in the local interstellar medium.
\end{abstract}

\section{Introduction}

Studies of interstellar deuterium Ly $\alpha$ are limited to sightlines with a total column density $\mathrm{N}(\mathrm{H} \mathrm{I}) \leq 18.5$, in order to avoid saturation of the deuterium feature by the hydrogen absorption. The potential advantages of using nearby hot WDs to study deuterium in the local interstellar medium have long been known (e.g. Lemoine et al. 1996). Although the modeling of the WD Ly $\alpha$ profile is not unproblematic, it can be performed with higher confidence than is possible for chromospheric emission lines. The WD continuum can be used to probe the velocity component structure by allowing observations of metal lines with small thermal widths. Finally, EUV observations of a hot WD can provide an independent estimate of the total H I column density.

STIS has three advantages over GHRS for such WD observations. The spectral resolution achievable with STIS $(\mathrm{R} \sim 170,000)$ is more than twice that of GHRS ( $\mathrm{R} \sim 80,000)$. The 2-dimensional echelle format of STIS allows $200 \AA$ to be recorded in a single spectrum, compared to only $6 \AA$ with GHRS. In particular, many important ISM lines, such as N I $\lambda 1200$, Si II $\lambda 1190, \lambda 1260$, $\lambda 1304$, and O I $\lambda 1302$ are obtained simultaneously with Ly $\alpha$. Finally, the twodimensional format of STIS should allow a more reliable correction for scattered light. Although the GHRS and STIS profiles show excellent agreement for typical interstellar lines (Howk \& Sembach 2000), the situation is less clear for a broad line profile such as Ly $\alpha$. The discrepancy noted by Sahu et al. (1999) between the interstellar parameters derived from GHRS and STIS observations of G191B2B is likely due to a slight shift in the GHRS continuum level across the $3 \AA$ of the profile (Sahu et al. 2000, in preparation). We currently have a STIS Cycle 9 program to study D/H toward the hot WDs GD 153 and HZ 43. 


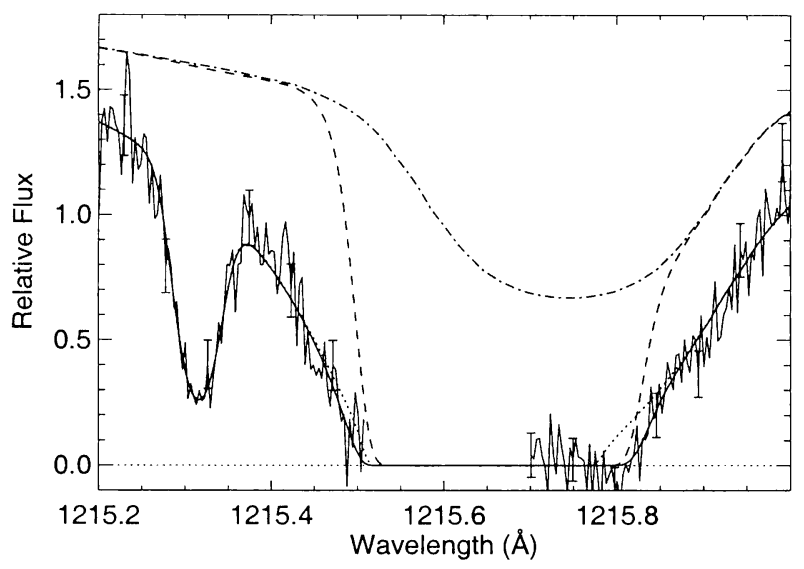

Figure 1. GHRS Ly $\alpha$ profile of HZ 43 and the model fit (solid line). Also shown are the non-LTE stellar profile (dot-dashed line), the hot hydrogen wall profile (dashed line), and the ISM profile (dotted line).

HZ 43 had been previously observed at modest S/N with GHRS by Landsman et al. (1995), who found that a good model fit was possible using a single ISM component, provided that one adopted a non-LTE Ly $\alpha$ stellar profile. However, as shown in Figure 1, a small discrepancy remains in fitting the wings of the saturated H I absorption, unless a hot $(\sim 20,000 \mathrm{~K})$, low-density $\left(\sim 10^{15}\right.$ $\mathrm{cm}^{-2}$ ) component is included, such as would be expected from the hot hydrogen wall surrounding the Sun (e.g. Linsky \& Wood 1996). Although there are large uncertainties in the derived density and temperature of the hydrogen wall toward HZ 43, the interstellar parameters are relatively well-determined, including both $\left.\mathrm{N}(\mathrm{H} \mathrm{I})=(8.9 \pm 0.4) \times 10^{17} \mathrm{~cm}^{-2}\right)$, and $\mathrm{D} / \mathrm{H}=(1.6 \pm 0.25(2 \sigma)) \times 10^{-5}$. The derived hydrogen column is in excellent agreement with EUVE results of Dupuis et al. $\left(1995 ; \mathrm{N}(\mathrm{H} \mathrm{I})=(8.7 \pm 0.6) \times 10^{17} \mathrm{~cm}^{-2}\right)$. The derived ISM temperature $(\mathrm{T}$ $=5700 \pm 800 \mathrm{~K})$ is more subject to systematic errors, but is in good agreement with the Orfeus results of Dupuis et al. (1998). The GHRS results on HZ 43 will be further discussed by Landsman et al. (2000, in preparation).

\section{References}

Dupuis, J. et al. 1995, ApJ, 455, 574

Dupuis, J. et al. 1998, ApJ, 500, L47

Howk, J.C., \& Sembach, K.R. 2000, AJ, in press

Landsman, W., Sofia, U.J., \& Bergeron, P. 1996, in Science with the Hubble Space Telescope - II, ed. P. Benvenuti, F.D. Macchetto \& E.J. Schreier (STScI), 454

Lemoine, M. et al. 1996, A\&A, 308, 601

Linsky, J.L. \& Wood, B.E. 1996, ApJ, 463, 254

Sahu, M.S. et al. 1999, ApJ, 523, L159 